\title{
The Constraint Interpretation of Physical Emergence
}

James Blachowicz

Loyola University Chicago, Jblacho@luc.edu

Follow this and additional works at: https://ecommons.luc.edu/philosophy_facpubs

Part of the Philosophy Commons

Author Manuscript

This is a pre-publication author manuscript of the final, published article.

\section{Recommended Citation}

Blachowicz, James. The Constraint Interpretation of Physical Emergence. Journal for General Philosophy of Science, 44, 1: , 2013. Retrieved from Loyola eCommons, Philosophy: Faculty Publications and Other Works, http://dx.doi.org/10.1007/s10838-013-9207-7

This Article is brought to you for free and open access by the Faculty Publications and Other Works by Department at Loyola eCommons. It has been accepted for inclusion in Philosophy: Faculty Publications and Other Works by an authorized administrator of Loyola eCommons. For more information, please contact ecommons@luc.edu. (c) $($ ) $\Theta \Theta$

This work is licensed under a Creative Commons Attribution-Noncommercial-No Derivative Works 3.0 License. (C) Springer Verlag 2013 


\title{
The Constraint Interpretation of Physical Emergence
}

\author{
James Blachowicz
}

\section{Loyola University Chicago}

\begin{abstract}
I develop a variant of the constraint interpretation of the emergence of purely physical (non-biological) entities, focusing on the principle of the non-derivability of actual physical states from possible physical states (physical laws) alone. While this is a necessary condition for any account of emergence, it is not sufficient, for it becomes trivial if not extended to types of constraint that specifically constitute physical entities, namely, those that individuate and differentiate them. Because physical organizations with these features are in fact interdependent sets of such constraints, and because such constraints on physical laws cannot themselves be derived from physical laws, physical organization is emergent. These two complementary types of constraint are components of a complete non-reductive physicalism, comprising a nonreductive materialism and a non-reductive formalism.
\end{abstract}

keywords: boundary conditions, constraint, emergence, non-reductive physicalism, reductionism 


\title{
The Constraint Interpretation of Physical Emergence
}

\author{
James Blachowicz \\ Loyola University Chicago
}

\section{Introduction}

In both early twentieth-century and contemporary investigations, the idea of emergence has rarely been evaluated on its own terms; rather, it's been used to support anti-reductionist accounts of life and mind. While this is an understandable strategy, it is also risky, for if genuine emergence is to be found even in purely physical (and chemical) phenomena, then it is unlikely that it will be sufficient or even decisive in explaining these more complex forms. In such circumstances, the idea can become quickly overburdened, and theorists may find themselves resorting to unhappy inventions to make it work. Or, working backwards, the need to have it explain these higher levels may lead these same theorists to deny that emergence is to be found at a purely physical level.

I want to argue that emergence is indeed found at this purely physical level, and further that it is not confined to exotic phenomena such as are found in quantum states, or even to novel chemical properties, but is in fact coextensive with all physical entities. The specific physical examples I have selected as vehicles for examining this idea, therefore, are as mundane as I can make them. This may lead some to think that the sense of emergence I have singled out is 
disconnected from that found in other theoretical discussions. I hope to show that this is not the case. $^{1}$

The basic principle underlying the version of the constraint interpretation of emergence that I will develop here is the non-derivability of actual physical states from the possible physical states represented in physical laws. Any physical constraint on a system is an actual state that acts to limit its degrees of freedom. The physical laws governing that system, on the other hand, only determine in general which physical states are possible for it.

There are two distinct types of physical constraint (and hence two distinct senses of "emergence"). The first type, which I will refer to as non-constitutive, would be acknowledged by any reductionist who agrees that no actual physical state can be derived from physical laws alone, that is, without the actual initial or boundary conditions that constrain those laws. These, typically, are constraints that describe non-essential properties of physical entities. The height from which I drop an object, for example, is a constraint on the law by means of which I may determine the time it takes to hit the ground. Reductionists, while granting this limited sense of non-derivability, would claim straightaway that it is trivial, and would not prove helpful in explaining emergence as that term is understood in the broader debate, where it describes the novel properties of complex, organized physical entities, such as are found in biology or even chemistry.

The second type of constraint, which I will refer to as constitutive (and which underlies a second sense of "emergence"), refers precisely to the non-derivability from physical laws alone of these more complex physical entities themselves (and not simply of the non-essential properties they happen to have). The very concept of a complex physical structure is incomprehensible, I will argue, without the concept of boundary conditions or constraints on 
physical laws. Complex structures are in fact sets of such interdependent constraints. I will argue that the concept of constitutive constraint can function as the basis for a (non-trivially) nonreductive physicalism.

To determine what grounds there may be for the non-reducibility of even simple physical entities such as atoms to their components and the physical laws governing those components, we must first understand the nature of the physical organization that constitutes a physical entity. At the very least, I will argue, such organization requires both a differentiation of structure and a relatively stable equilibrium that provides the basis for the individuation of the entity. As I shall explain, differentiation and individuation depend on two different species of constitutive constraint.

It should be clear that non-constitutive constraints cannot exist without constitutive constraints, for the former are non-essential properties of the physical entities constituted by the latter. That is, only fully differentiated and individuated physical entities actually exist.

I will first discuss constraint in general (common to non-constitutive and constitutive types), and then move to examine the constitutive constraints responsible for differentiated and individuated physical entities.

In defending a non-reductionist position, it should be clear that I will focus on the nonreducibility of physical entities and will consider the non-reducibility of properties only insofar as such properties are taken to be essential to the constitution of physical entities. I will only indirectly consider theoretic reduction, which addresses the question of the non-predictability of emergent physical states. 


\section{Actualizing Constraints}

\section{The Origin of the Constraint Interpretation}

The "constraint" interpretation of emergence was initially proposed by Polanyi (1968) and Pattee (1970), among others, and has since been adopted more widely, but not always with the same force or meaning. ${ }^{2}$ What made it an innovative approach to the problem of reductionism, of course, was that it focused on constitutive and not only non-constitutive constraint.

For Polanyi, "the morphology of living things transcends the laws of physics and chemistry" (1309) for the simple reason that such structures represent actual physical states defined by "boundary conditions" (also described as "initial conditions" and/or "constraints"); and boundary conditions are not derivable from the laws for which they form such conditions. As Polanyi put it, "a boundary condition is always extraneous to the process which it delimits" (ibid.). (He had presented an earlier version of this non-reductionist view in his Personal Knowledge, 1958/1964, 328--30). Boundaries are imposed on soup by a saucepan, on chess moves by a chess player, and on stone by a sculptor (1308). Simple, confining boundaries include those supplied by a scientist in a controlled experiment - for example, the angle of the slope of Galileo's inclined planes (1309). He distinguished (but not in a very principled way) non-constitutive "confining" boundaries such as these from the constitutive type that organize a material, evident in machines (1308).

The non-derivability of boundary conditions was acknowledged by Wigner $(1964,995)$, who described "initial conditions" as "the elements of the behavior [of physical objects] which are not specified by the laws of nature." However, Wigner found these conditions in the 
"irregularities" of nature rather than in ordered structures (996); that is, he apparently did not consider constitutive constraints.

Giere $(1968,410)$ responded to Polanyi's paper, arguing that "to describe this truth [the non-derivability of boundary conditions] by saying that the structure of the solar system transcends the laws of mechanics is at best misleading, for clearly the boundary conditions at $t$ do follow from the laws of mechanics provided only that we supply some other boundary conditions; for example the state of the system at $t$ '." Where Polanyi may have overvalued the nature of these conditions (they "transcend" the laws of physics), Giere seems to have undervalued them (we "only" have to supply additional conditions). Giere's view is a paradigm of the triviality objection. To acknowledge as he does that initial conditions themselves are not derivable from physical laws alone, that is, to admit the validity of emergence for nonconstitutive constraints, in no way eliminates the constraint interpretation of physical emergence as a basis for an account of constitutive constraint.

An organized system such as a biological organism or a machine, Polanyi observes, "works according to two different principles: its structure serves as a boundary condition harnessing the physical-chemical processes by which the organs perform their functions" (1308). A machine can break down, but "this will not affect the forces of inanimate nature on which the operation of the machine relied" (ibid.). Polanyi's use of the word "harness" here, unfortunately, does not address the nature of any distinctive causality, if there be any.

Polanyi draws his examples from artifacts (machines) and biological forms. He does not mention the organization of atoms or molecules. The only non-artificial physical example he considers is the sphericity of the Sun. He holds that this is entirely derivable from physical laws, 
maintaining that the fact that one cannot "observe" the behavior of the whole in the behavior of its parts does not constitute a case of irreducibility (1310). Yet one might argue that the Sun's sphericity is due to distinctive boundary conditions (including the proximity and extent of it parts) and is therefore not derivable from basic laws of physics in themselves.

Pattee $(1970,117)$ drew on Polanyi's analysis: "If you ask what is the 'secret' of a computing machine, no physicist would consider it any answer to tell you what one already knows - that the computer obeys all the laws of mechanics and electricity. If there is any secret, it is in the unlikely constraints which harness these laws to perform highly specific and reliable functions."

Sperry's $(1984,201)$ position on emergence appears identical. He observes:

... take a population of molecules, say, copper. You can shape this into a sphere, a pyramid, a long wire, a statue, whatever. All these very different things still reduce to the same material elements, the same identical population of copper molecules. Science has specific laws for the molecules, but no such laws for all the differential spacing and timing factors, the nonmaterial pattern or form factors that are crucial in determining what things are and what laws they obey. These nonmaterial space-time components tend to be thrown out and lost in the reduction process.

Wilson (2010) has recently offered a more refined treatment of the role of the loss of degrees of freedom in emergent physical entities. Although she does not discuss the constraint interpretation found in Polanyi, Pattee and others, she explicitly links loss of degrees of freedom to constraint. She also distinguishes the circumstances in which the degrees of freedom of the components of a physical entity may be reduced, restricted or eliminated. They are "reduced," for example, in the case of a "point particle constrained to move in a plane" (where one of its parameters is no longer free to vary but is held constant) (284--5). Rigid bodies (whose components are theoretically constrained in non-varying bonds) are another example of such 
reduction (286). A weaker case would involve, not "reducing" the degree of freedom in question to a constant, but "restricting" it to a range (285). Molecules, whose bonds are not rigid, are examples of such restriction (286). A weakly emergent physical entity, she argues, requires not only restriction or reduction of some degrees of freedom, but elimination. The laws governing macro-solids, for example, can completely ignore (in the "classical limit") the degrees of freedom represented by the spins of their quantum components (288).

Wilson insists both that all properties of weakly emergent entities are completely determined by lower-level component properties (this is what physicalism requires), and also that the elimination of some degrees of freedom of these lower-level components make the composing structure non-identical with and therefore ontologically non-reducible to its components - and thus "weakly emergent" (291--2). This is a clearer way of understanding the "harnessing" language used by Polanyi, Pattee and Sperry. This is also consistent with taking the "causal" action of constraints as essentially negative in nature: freedom of action must be eliminated to some degree for organized behavior to be possible. However, I think Wilson is too conservative in suggesting that it is only elimination (and not reduction or restriction) of degrees of freedom that qualifies physical entities as weakly emergent. I will return to this issue below.

McGivern and Rueger (2010) have recently argued that emergence can be found even in the simple case of a damped oscillator. An oscillator whose damping is not zero will spiral in on a singular value (a stationary state) from which it will not emerge, whereas one with no damping will oscillate continually. While they grant that there is a "sense of reduction" that applies when the equation for the undamped oscillator can be derived from the equation of the damped version (by setting the damping factor to zero), they insist that the solutions to the two equations exhibit 
a topological difference in their respective phase spaces that is qualitative (the differences are discontinuous) and that this is sufficient for us to judge that the differences are emergently novel and non-reducible one to the other (216--220). The damping is clearly a constraint on the oscillation that produces this qualitative difference. As to whether their example is too simple to qualify as an instance of physical emergence, the authors respond: "If the spectacular cases of emergence in phase transitions, solid-state physics, and quantum theory are bona fide instances of emergence, then they fulfill this role because they share the relevant features - novelty of behavior and non-reducibility on our sense - with our simple example" (220).

\section{Possible States and Actual States}

Suppose I drop an object out of a window on the third floor of my workplace, and I ask a physics student how long it will take to hit the ground. This student will certainly use Galileo's law to provide an answer, but only if an initial (or boundary) condition is supplied as well - the distance the object will fall. This may be a miserably trivial instance of non-constitutive emergence, but it does embody a necessary principle shared by more complex constitutive examples of the constraint interpretation. Even in this simple case, we can observe how a physical law acts as a bridge between certain actual states or values (the initial conditions) and consequent actual states. The law describes what is possible in any such circumstance, while the initial conditions describe which possibilities are actual (or will become actual). That is, it is actual states that provide specific values for the variables and parameters related in the laws. These values reduce the number of degrees of freedom expressed in these parameters. The reduction that such instantiation entails is the basis for the emergent character of the phenomenon. Compare Moreno and Umerez (2000, 104): "physical laws are just able to 
determine very basic and general features within the realm of the possible. But they still leave plenty of room for equally possible instantiations which might be actualized further due to factors ranging from mere chance to any kind of selective process, passing through weaker types of constraining influence."

This general perspective is reflected in Pattee's $(1972,250)$ observation that, while constraints require specific material embodiments, physical laws are "incorporeal." Salthe (1985, 70), in reviewing Pattee's conception, agreed that, without constraint, a natural process "could not produce anything"; "Nature would be, as it were, unformed" (72). This captures much of what Polanyi had intended in claiming that organized biological systems and machines “transcend" physical laws. Such "transcendence" implies no "trans-physical” principle, but rather the relatively simple transcendence of possible states by actual states (of variables by specific values). Nothing, of course, except perhaps pure possibility, is as ubiquitous as actuality; and so emergence in this general sense is a characteristic of physical existence itself.

It is important to realize that the non-derivability of actual states from possible states alone (determined by physical laws) without actual initial conditions itself relies on a more abstract principle - a principle that also underlies the non-derivability of possible states from physical laws without possible initial conditions. While Galileo's law may determine in general how long it would take an object to fall, given the height from which it is dropped, a single initial condition such as "forty feet" may be taken as one (non-actual) possibility. We can say, therefore, that it is impossible to derive how long it would take the object to hit the ground (hypothetically) from Galileo's law alone, that is, without the possible initial condition "forty feet." Yet this principle is even more abstract than this. Given the equation $x=1 / 2 a y^{2}$, where $x$ 
and $y$ are variables and $a$ is a constant, it is clear that we cannot derive a specific value for $y$ unless we first supply a specific value for $x$. This equation needn't have any physical interpretation (such as Galileo's Law) and therefore any reference to "actual states" of any kind for this to be true. That is, it is the distinction between variables and values that underlies the non-derivability of particular physical states (whether actual or only possible) from physical laws alone. Inserting a specific value for $x$ in this equation is in effect supplying an initial condition, but not, obviously, a physically actual one.

This being said, however, two additional points must be made. First of all, the kind of "possibility" represented by a candidate value (or hypothetical initial condition) is obviously a different kind from than that embodied in the specific relation among the variables of the equation (or physical law) ${ }^{3}$ itself. Initial conditions still function as values of variables, even if those values are taken hypothetically, while laws are relations among variables.

Secondly, if we hold that any empirical description of an actually existing physical state must be expressed as a complex set of specific values, while acknowledging that these values represent an instantiation of the variables of a physical law or laws, then the principle of the nonderivability of values from a relation among variables alone is equivalent to the principle of the non-derivability of actual states from possible states (expressed by a physical law) alone.

\section{Constrained Laws: Partially Actualized Possibilities}

While physical laws establish a range of physical possibilities which are constrained by actual states, these laws themselves result from more general constraints on even more general laws. Constraints specify values of open variables or parameters in a physical law. In Galileo's law, the parameter for the acceleration of a falling body will become a constant if we are 
calculating rates of fall only for objects falling to the Earth. This is clearly a more basic or general constraint on the law than the value of the distance from which an object is dropped. Laws of inertia and gravitational attraction may be described abstractly and with generality; but in order to describe a complex orbital planetary system where forces are balanced in a stable equilibrium, we need to constrain these laws with specifications of what were open parameters. A new "law" of planetary motion emerges only by means of these constraints. Kepler's First Law specifies that the orbits of the planets are ellipses; but this determination is a special case of that class of orbits that includes other conic sections. The First Law is a constrained version of this more general expression, where some degrees of freedom are lost. McGivern and Rueger's (2010) damped oscillator can be interpreted similarly. The existence of the damping factor itself in the equation for an oscillator constitutes a possible restriction on the range of its variation - an intermediate constraint of the type under discussion - while the specific value of that factor would constitute a more specific, actual constraint.

It is important to realize, therefore, that although a physical law expresses a range of possible states and is incapable of predicting any actual state unless some actual initial conditions are supplied, the law itself includes a limitation of possibilities at another level. Contemporary physics tells us that the basic physical laws we take as the foundation for any possible event within our range of observation are not, from another perspective, absolute. These basic laws, as well as the fundamental forces of nature, became differentiated in the first micromoments of the Big Bang. The earlier condition which they superseded, presumably, was significantly less constrained, allowing for wider possibilities than those permitted by these more differentiated laws. One may say, therefore, that any given level of a physical law, while 
representing possibilities that additional actual constraints will realize, also represents the product of actual constraints at a more fundamental level of wider possibilities, that is, more general laws. Being "more general" in this sense does not mean that these higher-level laws somehow exist as laws per se rather than as instantiations represented by specific values of events in space and time. These less-determined (less-constrained) physical states would simply represent more primitive, less organized stages of physical development.

Wigner $(1964,996)$ made a similar point with respect to what he called "invariance principles." These are "in a similar relation to the laws of nature as these [laws] are to events [initial conditions]." He finds these broader principles in very basic restrictions on the nature of space, time and motion (ibid.). If one is looking for the bottom-level possibilities that the most primitive constraints on the most primitive physical laws actualized, one would expect to find them in something like space-time itself and/or some other fundamental conception that physics has yet to characterize satisfactorily. The point is: the possibilities that specific physical laws embody are really intermediate forms - combinations of even wider laws and actual constraints - that may be further specified by more restrictive constraints.

This layering is also found in the constitutive constraints of physical entities. Where protons, neutrons and electrons provide the material for atoms, atoms provide the material for complex molecules. Atoms are formal organizational (constitutive) constraints for their material constituents; but they are also material constituents for the formal organizational constraints that constitute complex molecules.

One last point: While we may take physical laws to delimit possible states (given some actual initial conditions), and while we may also support the idea that only actual states exist, 
this does not mean that physical laws have no ontological "footing," so to speak. An actual physical state is not an atomic event disconnected from all other actual physical states: rather, an actual physical state is what it is as an instantiation of a physical law. Actual states, therefore, depend on the reality (if not the actuality) of partially determined possibilities. While the nonderivability of actual states from possible states is necessary for an ontology of emergence, the dependency of actual states on possible states provides a principle of unity that actual existence would otherwise lack - not the unity of an actual material stuff providing the components for physical entities (as a materialist reductionism might maintain), but the unity of a foundation of real possibility.

\section{Material and Formal Constraints}

The non-derivability of actual states from possible states alone, while necessary for any account of the "emergence" of either non-constitutive or constitutive properties, is not sufficient to explain the difference between non-constitutive and constitutive constraint and therefore the emergence of physical entities, which depends on the latter. To provide that explanation, we must identity what these constitutive constraints are. Put simply, we may ask: What is it about complex structure itself that depends essentially on boundary conditions and constraint?

It seems clear that in speaking of "actual states," we are speaking of states (properties) of material "things" and also of states that serve to constitute them. The only condition under which this would not be so, so far as I can see, would be if physical existence was possible apart from the existence of what would qualify as particulate entities. One might argue that space and time present themselves precisely as such non-individuated actual states. Yet there is some suggestion among physicists that space and time themselves may be particulate and/or quantized, and so this 
question may not be resolvable given our present knowledge of fundamental physics. Whichever way this is decided, constraints would still be necessary for explaining the complex structure that characterizes any particulate physical entity.

Physical entities, I would argue, must be both individuated and have differentiated structure. As I shall explain, individuating constraints may be taken as determinations of the material of which physical entities are composed, while differentiating constraints are determinations of the form this material acquires. This is not to say, however, that either nonindividuated matter or non-differentiated form actually exists, or that individuated matter and differentiated form can exist apart from one another.

What could qualify as non-individuated matter? Matter as purely homogeneous and continuous - perhaps in a fluid, ether-like form? While this might have been an option before the discovery of the microstructure of matter, the only obvious way for such apparently homogeneous, continuous matter to exist would be at more macroscopic scales as homogeneous, aggregated individuated particles. That is, individuation appears to be a necessary condition for the existence of any physical entity.

What could qualify as non-differentiated structure or form? "Atoms" do not exist generically, but only as carbon atoms, helium atoms, etc. The difference between two different kinds of atom is a difference in the number and arrangement of their specific components: only these fully differentiated species of atoms exist as actual individuals.

Extreme reductionism would refuse to take physical entities at face value. Whether we consider Democritus or Plato (or their contemporary descendants), what physical entities really are is apparently to be found in some pre-individuated or pre-differentiated condition. Given the 
distinction between material and formal determinations of physical entities, we can distinguish material reductionism from formal reductionism:

Material reductionism would take individuation as only a non-essential property of a primitive, non-individuated (or barely individuated), constitutive material. The more esoteric idea of formal reductionism would take differentiation as only an instantiation (a mere variation) of a more constitutive general principle. Opposing these reductionisms is the claim that neither non-individuated matter nor non-differentiated form actually exists. I will examine these two perspectives more completely in what follows.

\section{Individuating Constraints}

\section{Pluralization}

Actual, as opposed to possible, states of physical entities have specific coordinates in space and time. Space makes possible not only features such as location, size and amount of material, but more importantly, its pluralization - one entity here and another there (at the same time). Popper $(1953,107)$ provides a typical characterization of this rudimentary form of individuation: "Two qualitatively undistinguishable material bodies or bits of matter differ if they occupy at the same time different regions of space.” Pluralization is a feature of actual states (constraints), but not of the generalized possible states that physical laws describe. To specify plural components in any physical context is to supply initial conditions.

The pluralization of iterated individuals in space and time is a key element in a constraint theory of the emergence of physical entities. Without it, no components would be available from which any physically organized entity could arise. Physical laws by themselves are incapable of 
specifying the exact numbers, kinds and configurations of these actually existing pluralized components, for these specifications are themselves initial conditions for those laws. This is not simply a case of the non-reducibility of non-constitutive constraints or boundary conditions from the laws which they constrain - a rudimentary form of emergence dismissed as trivial by reductionists - but the non-reducibility of the constitutive constraints that define any physical entity. That is, no physical organization (and hence no physical entity) can be explained by physical laws alone.

Individuals as Discrete Sets of Interdependent, Stable Constraints

Transient and disordered aggregates fail to qualify as individuals, and therefore give rise to no appreciably new (emergent) unified physical entities. Wimsatt (1997), in order to determine what does not qualify as emergent, examined the properties of aggregates. He pointed to the conservation laws (of mass, energy, momentum, net charge) as most likely the only characterizations we can make of such relatively constraint-free collections. He concluded that "very few system properties are aggregative, suggesting that emergence, defined as the failure of aggregativity, is extremely common.” (S382).

What shall we add to disordered aggregates to be able to speak of emergent physical entities? We will need a more complex form of constraint - one that arises from a set of interdependent components.

The specific height from which I drop an object qualifies as an initial or boundary condition (a non-constitutive constraint), and the fact that I cannot derive from Galileo's law the time it takes that object to hit the ground without supplying that initial condition will hardly 
impress anyone seeking to understand the emergence of physical entities. Suppose we add more conditions:

First, we should probably have included another condition from the start, namely, the constant rate of acceleration due to Earth's gravity. Secondly, since we are dropping an object through the air, its fall will be resisted and we will have to factor in its density, as well as characteristics of the air, including its temperature and atmospheric pressure. Suppose now that we observe that the ground beneath the window is significantly sloped. While this condition will not affect our prediction of the time it takes for the object to make contact with the ground, it will affect how the object behaves subsequent to that contact. The object may embed itself if the ground is muddy; or, if the ground has a hard surface, the object will tend to bounce, most likely, downhill.

Each of these other conditions is relatively independent of the others, that is, each one could be varied while keeping the others constant. A new situation arises, however, when we consider interdependent conditions. The object itself represents such a set of conditions, that is, a set of constraints that constitute that physical object in a primitive way. Different shapes will make the object more or less aerodynamic, and will also determine its behavior once it hits the ground. The shape is formed by adjacent positions of the surface particles, each particle acting as an individual, constrained element. It is not simply the initial positions of all these particles relative to one another that is significant here, but the fact that they are also fixed relative to one another and relatively resistant to disruption. Such a collective stable constraint, which reduces the degrees of freedom of its components and delimits the discreteness of the set as a whole, appears to be a necessary condition for the individuation of physical entities. 
Pattee $(1970,125)$ observed that, in physical organizations, "some particles join together in a more or less permanent collection, otherwise the 'collection' would only be transient and would depend crucially on the initial conditions." That is, he distinguishes transient or contingent initial conditions from those that characterize a stable equilibrium of components. Thus, he describes a "constraint" as the "permanent loss of degrees of freedom of collections of matter in solid bodies" (126).

The actual things of nature are "things" in part because they each have an appreciable enduring identity through time, resisting disruption and disturbance while also, because of their lifetimes, being able to join with other entities in larger stable structures that may qualify as new entities. It is with structures composed of multiple parts, of course, that one commonly finds stable equilibria, for in such structures, a balance can be struck among these extended parts. One might even find in this fact some reason for supposing that no physical entity can exist without an equilibrium of such parts. Kant wisely refused to take the endurance of even a noncomposed particle of matter as a given fact (as he believed mechanism did). Rather, he held that material particles represented a balance (a stable equilibrium) between repulsive forces that threaten to expand the particle without limit and attractive forces that threaten to collapse it to a point. ${ }^{4}$

Stable equilibria often function as attractors in physical systems. That is, they are states that a system may tend to enter and remain in, regardless of the system's initial state. Muir $(1982,127)$ in fact described these attractors as constraints themselves. When the parts of a stable structure "join together," as Pattee put it, it is attractive physical force that secures the stable equilibrium. Attractive force is the workhorse of constraint and an apparently necessary basis for the reduction of degrees of freedom defining any physical entity. While solid entities 
eliminate disruptive motion of their constituents (within certain bounds), a motion can still be constrained by forces without being eliminated in this way, as is evident, for example, in gravitational systems, which are by and large dynamically stable. This is not to say that gravitational systems qualify as full-fledged, emergent physical entities (this is an issue, however, that is outside the scope of the present discussion).

\section{Differentiating Constraints}

Those who have discussed individuation in both historical (ancient and medieval) and contemporary contexts have often distinguished between the sense in which an individual is taken to be simply a discrete, stable item in space, distinct from other such items, from the sense that characterizes a true physical entity, which includes additional conditions that determine the unique properties that distinguish one such entity from another of a different kind. Thus Popper's reference to "bits of matter" appears to make no assumption that such an individual is itself an organized unity whose identity is a product of its differentiated organization. Bits of gold sliced off from a larger sample become plural instances; but no claim of organizational unity is made for each bit insofar as it is a bit (i.e., an aggregate), although the same cannot be said for the individual elementary particles of which the bit is composed. Each of these plural bits is an instance of matter; they constitute this plurality regardless of whether they were gold or silver or copper. What makes each bit gold and not silver is determined by its distinctive differentiated microstructure, not by its discreteness and stability as a bit.

A bit of matter has the appearance of individuation because it possesses the stability and discreteness that true individuals require. Its molecules are constrained, having their degrees of 
freedom restricted. When the object is thrown, its parts move as a group. One might well argue that the mere fact of its solidity qualifies this form as an organization exerting "downward causation" over its parts.

Yet a homogeneous solid of this sort lacks the integrated, differentiated organization of its components (with distinctive properties and functions arising from that organization) that characterize non-aggregated physical entities like a water molecule or an organism. Unlike complex, differentiated entities, homogeneous solids can be fragmented without affecting any of the important properties that characterized the unfragmented whole. In this respect, an aggregated solid is a pseudo-entity.

To become genuinely individuated, various multiple, individually simple pieces or functions must be put together in different stable arrangements, yielding a differentiated structure that enables the whole to behave in very specific ways - ways that constitute its distinctive properties. Such true individuals do not retain their nature if fragmented and reduced to bits.

It is true that because actual constraints (here-and-now restrictions of degrees of freedom) are not derivable from physical laws alone, and because a solid bit of matter requires such constraints, it follows that a solid bit of matter is not derivable from physical laws alone. Yet because such a bit is really an aggregate, such "emergence" is non-constitutive and does not yield an individuated physical entity.

\section{Formal Science}

The special class of constraint represented by differentiated organization opens up the world of attractors that Emmeche et al. $(2000,27)$ thought deserving to be treated as "Platonic" 
in some way. These attractors are potential formal, differentiated organizations; they "exist" before they are realized, "just as water - given the properties of oxygen and hydrogen - existed as a potentiality, as an attractor, before the single empirical water molecule was realized" (29).

This suggests the possibility of a kind of Platonic "science" of possible forms of differentiated organization - a purely formal science of complexity that would be distinct from material physics. Cybernetics, information theory, the theory of computation and complexity theory have all in different ways contributed to this expectation. In A New Kind of Science (2002), Stephen Wolfram made the claim that the computational theory of cellular automata represents a more fundamental way of understanding the world than does primary physics (relativity theory and quantum theory). In his review of that book, the physicist Stephen Weinberg (2002) pointed out that this view had surely not been conclusively established by Wolfram, but admitted that, in fact, it might one day be shown to be correct. This would divide, as it were, the natural sciences into sciences of matter (basic, unified physical laws) and sciences of form (theories of complex, differentiated organization).

The reductionism that emergence theory has typically struggled to avoid is materialist in nature, where very fundamental physics threatens to swallow up all the non-fundamental special sciences, and where some very primitive material (even a non-differentiated "stuff") is taken as the ontologically constitutive principle of all more complex organizations of matter - much in the way that all objects made of wood are taken to be, really, just wood.

Yet there is another form of reductionism that threatens an equally devastating collapse but pulling from the opposite direction - where all sciences would be reduced to some kind of basic "laws of form." 


\section{Formal Reductionism}

Suppose we were able one (very distant) day to characterize all of the formal types of organizations that might be actualized in nature. Terrestrial biology would be only one material example of the possible biologies that such an organizational science might delimit. One might be tempted in such a formal science to look for some relatively simple foundational principle (or principles) from which, in theory, all more complex organization could then be derived. There is something of this expectation found in the focus on recursive algorithms (such as in Wolfram, 2002) or in the "game of life," an example offered by other investigators, such as Chalmers, 2006), where simple transformations, repeatedly applied to resultant states, produce forms or functions that are judged to be surprisingly more complex.

Specific or differentiated formal organizations might then be reduced to variations of some smaller set of more general forms, and these to even more general forms, rising perhaps, to some most general proto-form or formal operation. This ambitious expectation might even use as positive evidence the fact that, after all, complexity increases naturally in the universe, and so the more differentiated (more specific) formal structures must be derivable from the simpler (more general) forms.

This species of reduction yields consequences that are not really very different from those of a materialist reduction and, I would argue, should be rejected on the same grounds.

A materialist reduction reduces individuated entities to non-individuated matter (a "stuff" common to all physical entities) and to general physical laws. The constraints that account for the individuation of such entities are treated simply as (trivial) properties of the common stuff and nothing substantive in themselves. The constraint interpretation, on the 
contrary, would hold that the individuating, constitutive constraints on the common stuff and/or the physical laws that govern this stuff, because they are not derivable from this stuff or these laws themselves, qualify as emergent physical entities. Individuation is explainable only through the specification of types and numbers of an individual's components - a specification that represents crucial initial conditions not derivable from physical laws alone. The constraint interpretation would find this emergence wherever any type of individuated physical entity is identified - in protons no less than in living organisms.

A formalist reduction reduces differentiated structure to general formal principles common to all structures. But general formal principles are not sufficient to account for their more differentiated actual variants for the same reason that the possibilities delimited by physical laws are not sufficient to account for individuated actual states. Like individuating constraints, the differentiating constraints that specify more general forms require determinations not derivable from the general forms that they differentiate. "Atom" is a general form (a genus); to specify "atom" as "carbon" or "hydrogen," one must supply a differentia ( so many protons, neutrons, electrons organized in a certain way). Such a differentia is not logically derivable from the genus, for a differentia is, in effect, a logical constraint on a genus, reducing, one may say, the degrees of freedom permitted by the genus.

If we were to hold that these specifications of the general were "mere variations" of it, and that it is the generality of the form that is its constitutive principle, there would be nothing to prevent a reduction of all complex forms to one most general form or formal principle. This would be the formal equivalent of a materialistic reduction: in the latter, we take actual, individuated states to be "mere" instantiations of physical laws (reducible to them); in the former 
we take actual, differentiated organizations to be "mere" instantiations of more general laws of form (reducible to them). ${ }^{5}$

While it is true that we may think of formal structures abstracted from physical embodiment ("software' as distinct from "hardware") as general types relative to specific types, and thus seemingly free of the constraining conditions of actual material existence, even abstract formal structures must employ the iterative operations that require a plurality of components. These complex, organized iterations are ipso facto constraints that negate alternative iterations. That is, formal differentiation acts as a boundary condition or constraint no less than does material/physical individuation, and cannot be derived from the more general principles it constrains.

In summary, then, I would argue that neither individuation nor differentiation, which I have proposed as criteria for any physical entity, can be explained by or reduced to physical laws alone. The reason is that individuation and differentiation require a here-and-now specification of the number, kind and arrangement of a physical entity's components: For example, this number and these kinds of atoms - two hydrogen, one sulfur and four oxygen - arranged in this way and not that, to yield an emergent sulfuric acid molecule. These specifications are constraints or boundary conditions on physical laws. This is not the trivial non-derivability of non-constitutive constraint, but the genuine emergence of the constitutive constraints defining physical entities. It is the basis for a non-reductive physicalism that is coextensive with all physical entities, from protons (at least) through living and thinking organisms. Artifacts: Superficial Macro-Differentiation 
If artifacts are individuated and differentiated, do they qualify as emergent physical entities?

Artifacts are layered at least twice over. A natural physical object like an atom is an organization of differentiated parts. Yet when combined, atoms and molecules may form larger stable structures in relatively homogeneous solid materials - which can then provide moldable components that are artificially differentiated. In a macro-object like a clock, there are two organizational layers: a micro layer with micro-organizations of micro-particles - for example, copper atoms organized out of differentiated sub-atomic particles - and a macro-layer with a macro-organization of macro-particles - the clock organized out of differentiated copper parts. What separates these two layers from one another is the homogeneous material (solid copper macro-material that is molded to produce the differentiated macro-parts).

The organizational structure at the macro-level of an artifact does not utilize the differentiation of the micro-parts (except insofar as they provide overall macro-properties of the homogeneous material), but utilizes only the artificial differentiation of the macro-parts. The differentiation of these macro-parts is imposed on the intermediate homogeneous material. What is thus artificial results from what is superficial. An artifact is, as it were, a "superfact" - an object created by molding the surfaces of other objects and using these superficially differentiated objects as parts. The homogeneous layer insulates the differentiated microstructure from the differentiated macro-structure.

My inclination is not to take artifacts as true physical entities, for the intermediate homogeneous layer destroys the unity that characterizes a physical entity. Such a homogeneous layer qualifies as an aggregate rather than as a genuinely differentiated structure. This is true 
even if the components of the aggregate are differentiated: a rock is still an aggregate even if its components and their distribution (including features such as porosity) are diverse. Smashing a rock into smaller pieces produces only smaller rocks; this is not the case with a truly differentiated entity such as a single complex molecule or an organism.

At most, the artificial unity of the superficial layer may be taken as an emergent property of the physical entities composing the homogeneous material rather than as an emergent physical entity in its own right; but this is a discussion I cannot pursue here. ${ }^{6}$ Are Atoms and Molecules Physical Entities?

Since my concern has been primarily to defend a type of pan-physical emergence, I have not provided any detailed taxonomy of kinds of physical entities. Yet I would not want to give the impression that the only emergent physical entities are some ontological "simples" to which all more complex (apparent) "entities" would be reduced. On the contrary, I would hold that the principles of individuation and differentiation are criteria that identify physical entities throughout the physical hierarchy of material organization - sub-atomic particles, atoms, molecules, living organisms (at least). Yet there is not widespread agreement as to whether atoms and molecules qualify as physical entities.

The stable equilibrium which is a minimal requirement for individuating a physical entity is evident in molecular and atomic structures and even in particles at the sub-atomic level. The balance of forces in such structures is not imposed from without, as is the case with artifacts held together by the introduction of fasteners of some kind; rather, the balance is a natural effect of the forces exerted by the components themselves. The equilibrium, we may say, is internal to the entity. Further, the differentiation of the components of these structures determines the entity's 
distinctive properties (carbon vs. hydrogen, etc.). I would argue, therefore, that molecules, atoms and at least some sub-atomic particles qualify as genuinely individuated and differentiated physical entities.

Pattee $(1972,250)$ apparently agrees, affirming that a "constraint . . must have some distinct physical embodiment in the form of a structure" and mentions "molecules, membranes, typewriters, or table tops" (see also 1970, 165). (Here he is overly generous, I think, in granting physical entity status to artifacts as well as molecules.)

On the other side, Wilson (2010, 307--8), for example, is reluctant to have molecules qualify as emergent physical entities, in part on the grounds that they do not eliminate, but only restrict the degrees of freedom of their components. Yet if a degree of freedom is restricted to so small a range that it can effectively be ignored (that is, treated as eliminated), the argument for ontological non-reducibility would not necessarily be weakened, as Wilson fears.

Peter van Inwagen (1990) also appears to deny the status of physical entity to atoms and molecules - largely, it appears, because in his view they fall short of the "self-maintaining" (or what might better be called "self-reconstituting") processes found in living organisms. He first uses the analogy of a social club whose members are leaving and joining in equal numbers, so that what remains is the club's "form" or internal constitution (84--85). He then conceptually converts this model to a system made up of automata, using "new" members only for their parts (85). This is a self-maintaining phenomenon or "homeodynamic event"; but unlike other such self-maintaining events, like a wave, a candle flame or a storm (such as Jupiter's Great Red Spot), it is what van Inwagen calls a life - "well-individuated' and "self-directing' (87). 
He insists that any true physical entity must be either such a self-maintaining life or a "simple" (non-decomposible) particle (a quark or an electron) that become the parts for such a life (158). Tables (and almost all other artifacts), as well stones and stars (aggregates) are not true physical entities, for they lack the characteristics of self-maintenance and individuation. This view seems to entail a rejection of the idea that protons, atoms and molecules qualify as true physical entities.

Before he comes to discuss life, van Inwagen does consider what other sorts of "composition" might lead to a physical entity, examining "contact," then "fastening," "cohesion" and "fusion" (33--61). These appear to him as crude attempts to take material objects as static, spatial juxtapositions of matters - thereby failing to focus on the thing's interior principle of activity. Yet van Inwagen does suppose that an artificial form of a self-maintaining, individuated physical entity may be possible - and therefore may qualify as a life (137). I would claim, on the contrary, that the division between an artifact's microstructure and macrostructure may prevent the unity necessary for a genuine physical entity.

Would van Inwagen take the composition of an atom to be on a par with the composition of a stick-model of an atom? While a chair may be "simples arranged chair-wise," would an atom be "simples arranged atom-wise"? Unlike an artificial model, the balance of forces in an atom is not imposed from without, but is a natural effect of the forces exerted by the components themselves. Its equilibrium is internally generated. Further, the differentiation of its components determines its distinctive, quite individuated and dynamical properties. An atom is neither parts externally fastened together, nor a non-individuated 'storm" of activity. Nor, at a higher level, is 
a sodium chloride molecule a kind of pair of Siamese twins. Such twins do not possess a unified identity or principle of activity the way a chemical compound (or an atom or a proton) does.

I'm not sure why van Inwagen doesn't say more about atoms and molecules. All of his examples of non-artificial inanimate objects seem to be taken from macro-world aggregates (stones, mountains, stars). He does say that there are "no visible inanimate objects" (213). Does he intend "visibility" as a criterion? We are certainly not restricted to the visible spectrum, and one would think that images from an electron microscope would count. Yet perhaps I am wrong in thinking he meant to take macro-visibility to be a criterion.

To suggest that an atom is an "arrangement" in the way that an artifact or a natural aggregate such as a rock is an arrangement doesn't do justice to the way in which an atom utilizes the natural differentiation of its components. The parts of atoms have neither the plasticity nor the arbitrariness we associate with the parts of artifacts or shaped aggregates. A single proton, single neutron and single electron combine in a stable arrangement in only one way (as a deuterium atom). While one might speak of assembling atoms into different kinds of molecules, this assembly is highly constrained by internal forces and stability considerations; the parts of a brass clock, on the other hand, can be reassembled into a wide variety of forms with very few constraints of this type.

Van Inwagen does not discuss "emergence" as such, but does mention "holism," which he characterizes as "the thesis that the properties of organisms are not wholly determined by, do not wholly supervene upon, the properties of their parts" (90). He explains further in saying that “even a complete and correct list of principles of composition would not enable a perfect reckoner - the Laplacian Intelligence, say - to reckon the properties of wholes from the 
complete truth about the intrinsic properties of and all the relations that hold among the parts that compose the wholes" (ibid.). But I have argued that, as long as one includes the initial conditions that specify the "relations that hold among the parts," there is no obstacle in principle to deriving a knowledge of the resultant whole. Yet van Inwagen acknowledges that he doesn't know whether holism is correct; and that, besides, either way, it would not affect his theory.

Apart from the status of atoms and molecules (and the nature of the "simples" out of which organisms are composed), van Inwagen and I more or less agree with Aristotle on the criteria of individuation and differentiation, as well as on an emphasis on active functioning and not just on materiality. He notes, in fact, that his view of physical entities is closest to Aristotle's, differing only on the subject of metaphysical atomism. Yet Aristotle acknowledged that it was to Democritus' credit that he at least preserved the individuality of his atoms, and thereby refused to reduce substances at this level to some homogeneous, non-individuated material. Had Aristotle been an atomist, he would have balked at treating atoms as nondifferentiated entities with no "inner" life or dynamic natures. Given our contemporary picture of them, however, I think he (unlike van Inwagen) would have welcomed them into the world of genuine substances.

\section{The Ontology of Emergence}

Both critics and defenders of emergence tend to see the constraint interpretation as too simple to provide an account of qualitative novelties, especially those found in biological and mental phenomena. Many would maintain this negative appraisal even with the elaboration provided by the principles of individuation and differentiation underlying constitutive constraint 
I have proposed. The defenders have often felt the need to appeal to something more drastic to make their case - to something trans-physical - in the form of substance dualism, or property dualism, or causal dualism or some combination of these.

\section{Critics of Emergence}

Few would deny that a given physical state can be explained by supplying the various physical laws that pertain as well as the initial conditions for those laws. If a necessary basis for emergence is the non-derivability of this physical state from these laws alone, there would be little or no reason to suggest that such emergence introduces non-physical principles. To this extent, emergence is safe from reductionist critics who would claim otherwise.

Yet as I have already pointed out, these same critics may then claim that such nonderivability is a trivial matter, with no significant ontological implications. Baylis (1929) provided an early form of such a criticism. He agreed that emergence is in fact ubiquitous, but that this indicates that "it solves none of these problems [e.g., evolution, secondary qualities, mind-body, mechanism/vitalism], supports no one Weltanschauung rather than any other, and does not even imply evolution" (372). The "ubiquitous" form of emergence Baylis singled out was that described by E. B. Spaulding in America and by C. D. Broad in England, who each proposed that an organized whole was emergent for the simple reason that it possessed properties not possessed by its parts (see Blitz, 1992, 86, 118). There are two aspects of the examples Baylis considers, however, that reveal a difference between the "ubiquitous" emergence he criticizes and the version I have defended.

First of all, he notes that properties that may be taken as qualitatively emergent in an organized whole, such as the peculiar properties of $\mathrm{H}_{2} \mathrm{O}$, "may now be discovered to be 
properties of the electronic constituents of some of these atoms" (377). Baylis is here playing on the supposition of many emergentists that such properties could not in principle have been predicted from the properties of the parts (and their organization). This is not a supposition I have made. I would defend the reverse: that the properties of higher organizational levels can indeed (sometimes even in practice and not only in principle) be anticipated from a knowledge of their components together with the constraints that describe their organization - but that nevertheless the stable equilibria that characterize these organizations do constitute genuinely new physical entities (because these constraints cannot be derived from a knowledge of the components alone).

This view is quite close to Wilson's (2010), which supports the physicalism of nonreductive physicalism by endorsing the theoretical derivability of laws treating "weakly emergent entities" from the laws of more fundamental physical entities, and yet also supports its non-reductive character on the grounds that these emergent entities have eliminated "degrees of freedom" present in the lower-level entities and are thus not identical with them (298--303).

Secondly, Baylis includes in organized "wholes" examples that I would exclude: moving a book from the second to the third shelf of a bookcase would presumably provide a new "emergent" whole: "The complex constituted by the color pattern of the books formerly on the second shelf is destroyed and the complex constituted by the color pattern of the books now on the third shelf is formed" (376). I have argued, however, that such initial conditions are nonconstitutive and indeed do not account for emergent physical entities. Genuinely emergent physical entities must also comprise stable equilibria with differentiated structure. The bookpattern example qualifies as an aggregate, not a physical entity. And so, while I do believe that 
emergent physical entities are ubiquitous, they are not quite as universal as Baylis' conception would suppose.

Chalmers (2006) provides a more contemporary slant on the same question, but his account suffers from the same defect. He distinguishes "strong emergence," which holds that properties of emergent wholes are not deducible from the properties of their parts (he has consciousness in mind), from "weak emergence," for which such non-deducibility is not a requirement. He rejects the idea that weak emergence depends simply on the idea that systems possess properties that their parts lack, for, on this view (he says), even filing cabinets and packs of cards would be emergent (251). He does not examine in any detail the constraintinterpretation of emergence or the elaboration of that interpretation I recommend where constitutive constraints yield emergent actual physical entities that are individuated and differentiated; nor does he offer any example of physical emergence that might be found in simple chemistry (or in artifacts). Rather, he focuses on the idea that a "weakly emergent property of a system is an interesting property that is unexpected, given the underlying principles governing the system" (253). The recursive application of simple principles (such as those that comprise the "Game of Life"), he says, could provide such unexpected results. While in general this is an interesting category of organizational theory, it fails to recognize how essential are the principles of individuation and differentiation I have outlined.

Where Baylis' account of emergence as "wholes greater than the sum of their parts" failed to acknowledge the utility of the idea of constitutive initial conditions or constraints, C. Lloyd Morgan $(1923,16)$, whose theory of emergence Baylis was criticizing, did seem to recognize its role: “ ... when some new kind of relatedness is supervenient ... [on lower levels], 
the way in which the physical events which are involved run their course is different in virtue of its presence." To be sure, Lloyd Morgan specifically had living organisms in mind, and he may not have acknowledged such "supervenience" in the case of purely physical organization, but the idea that it is not just some overall "pattern" or juxtaposition of parts that constitutes emergence, but rather the constitutive constraints that restrict lower-level events, reducing their degrees of freedom, is a more nuanced view that Baylis seems to miss.

Critics of emergence will often enough refer to "organization," "configuration," "pattern" or "form" - but without ascribing to this aspect of things any special status. Klee $(1984,45)$, for example, while conceding that Polanyi's reliance on boundary conditions in characterizing emergence is "coherent," questions "the need to treat these [conditions] as something other than relatively complex properties." He also questions whether, if such properties are predictable from an understanding of lower levels, one should speak of emergence at all (46). Klee initially proposed that emergence not be understood solely in terms of an "increase in complexity of structure," for he holds that such a view is "wholly compatible with the micro-determination of higher-levels of organization by their lower-level microparts" (47). I would argue that Klee thereby fails to appreciate how “complexity of structure' entails constraints on those lower-level microparts, and is thereby not determined by them alone.

Klee also holds that, in the case of Sperry's wheel, we should not think that it is a macrostructure determining the micro-structure (a kind of "downward causation"), for the influence exercised by the wheel as a whole on its molecules occurs through "structural micro-connections with molecules in the wheel" (61). He does acknowledge, however, that "this is a kind of determination that is 'holistic' insofar as the effect is transmitted to the molecule through a 
larger holistic micro-structure" (ibid.). He insists, however, that this holistic micro-structure "does not seem ultimately to be a kind of determinative influence that can't be given a basically micro-structure mechanism" (ibid.). Besides, he says, the wheel is only a metaphor (something Sperry denied); macro-determination, he suggests, is better considered in the sorts of biological examples Donald Campbell and others provided.

The issue of the nature of the influence of macro-structure on micro-structure is relevant to a more comprehensive understanding of emergence, but real emergence is a phenomenon that may be observed even in a simple molecule - which Klee would classify as only a microstructure. And so we are back to the distinction between micro-parts and the micro-whole that constitutes the constraints on them.

\section{Defenders of Emergence}

Many early emergentists appeared to agree that mere physical "complexity" of an organized whole would be insufficient to account for novel properties. They thereby unfortunately embraced one of the principal tenets of reductionism. Because these defenders held that novel properties and/or entities did emerge, they had to invoke other sources for this novelty.

My principal concern with this strategy is that, by relying on more than a constraintbased ontology, they leave themselves open to reductionist attacks that threaten to invalidate the idea of emergence entirely - or to confine it within the ramparts of the philosophy of mind. The ubiquity of physical emergence has led both sides to either underestimate its significance or even miss it entirely. In a way, the reason it has been so hard to characterize emergence is that it is so easy to characterize it. 
Although not exactly parallel to my own, Paul Humphreys' (1997) view of emergence does not suffer from this defect. He agrees that "emergent properties are probably quite common in the physical realm" (S344), but he wisely judges that "this will require refinement in terms of what we mean by physicalism" (S345). He also correctly observes that the presence of emergence at the purely physical level "dissolves the air of mystery that has traditionally surrounded emergentism and has led clear-headed philosophers to stay away" (ibid.).

Such a pervasiveness is exactly what is needed to reconfigure our ontology of physical nature. A conception of emergence that is nature-wide is a safeguard against the various dualisms that have resisted real explanation. Yet what I have proposed also relies on two fundamental dualisms of a more basic type: that between possibility and actuality; and that between individuation and differentiation - in effect, between matter and form. 7 


\section{References}

Andersen, P. B., C. Emmeche, N. O. Finneman, P. V. Christiansen (Eds.) (2000). Downward causation: Minds, bodies and matter. Aarhus: Aarhus University Press.

Baylis, C. A. (1929). The philosophic functions of emergence. Philosophical Review, 38, 37284.

Beckermann, A., H. Flohr and J. Kim (Eds.) (1992). Emergence or reduction? Essays on the prospects of nonreductive physicalism. Berlin: Walter de Gruyter.

Blachowicz, J. (2012). Essential difference: Toward a metaphysics of emergence. Albany, NY: SUNY Press.

Blitz, D. (1992). Emergent evolution: Qualitative novelty and the levels of reality. Dordrecht: Kluwer.

Broad, C. D. (1925) The mind and its place in nature. London: Routledge \& Kegan Paul.

Chalmers, D. J. (2006). Strong and weak emergence, in P. Clayton and P. Davies (Eds.) The reemergence of emergence (pp. 244-256). Oxford: Oxford University Press.

Christiansen, P. V. (2000). Macro and micro-levels in physics, in Anderson et al. (Eds.) Downward causation: Minds, bodies and matter. Aarhus: Aarhus University Press, pp. $51-62$.

Corradini, A. and T. O'Connor (Eds.) (2010). Emergence in science and philosophy. London: Routledge.

Emmeche, C., S. Køppe and F. Stjernfelt.(1997). Explaining emergence: towards an ontology of levels. Journal for General Philosophy of Science, 28, 83-119. 
Giere, R. N. (1968). Structure of an organism, Science, 162, 410.

Humphreys, P. (1997). Emergence, not supervenience. Philosophy of Science, 64, (proceedings), S337-S345.

Kant, Immanuel. (1970) [1786]. The metaphysical foundations of natural science, translated and introduction by James Ellington. Indianapolis: Bobbs-Merrill.

Klee, R. (1984). Micro-determinism and concepts of emergence. Philosophy of Science, 51, $44-63$.

McGivern, P. and A. Rueger (2010). Emergence in physics, in A. Corradini and T. O'Connor (Eds.) Emergence in science and philosophy (pp. 213-232)

Moreno, A. and J. Umerez. (2000). Downward causation at the core of living organization, in Andersen et al. (Eds.) Downward causation: Minds, bodies and matter (pp. 99-117). Aarhus: Aarhus University Press.

Morgan, C. Lloyd. (1923). Emergent evolution. London: Williams and Norgate.

Muir, A. (1982). Holism and reductionism are compatible, in S. Rose (Ed.), Against Biological Determinism (pp. 122-135). New York: Allison and Busby.

Pattee, H. H. (1970). The problem of biological hierarchy, in C. H. Waddington (Ed.) Towards a theoretical biology: 3: Drafts (pp. 117-136). Chicago: Aldine Publishing Co.,

Pattee, H. H. (1972). Laws and constraints, symbols and languages, in C. H. Waddington (Ed.), Towards a theoretical biology: 4: Essays (pp. 248-250). Edinburgh: Edinburgh University Press. 
Polanyi, M. (1958/1964). Personal knowledge: Towards a post-critical philosophy, revised edition (1962) published by University of Chicago Press. Harper Torchbook edition (1964) New York: Harper \& Row.

Polanyi, M. (1968). Life's irreducible structure. Science, 160, 1308-1312.

Popper, K. (1953). The principle of individuation. Aristotelian Society Proceedings, Suppl. Vol. 27: Berkeley and modern problems (pp. 97-120).

Salthe, S. N. (1985). Evolving hierarchical systems: Their structure and representation. New York: Columbia University Press.

Salthe, S. N. (1993). Development and evolution. Cambridge: Cambridge University Press.

Sperry, R. W. (1984). Emergence, in P. Weintraub (Ed.) The Omni interviews (pp. 187-207). New York: Ticknor \& Fields.

Stephan, A. (1997). Armchair arguments against emergentism. Erkenntnis, 46, 305-314.

Van Gulick, R. (1993). Who's in charge here? And who's doing all the work?, in J. Heil and A. Mele (Eds.) Mental causation (pp. 233-256). Oxford: Oxford University Press.

van Inwagen, P. (1990). Material beings. Ithaca, NY: Cornell University Press.

Weinberg, S. (2002). Is the universe a computer? The New York Review of Books, October 24, 2002, pp. 43-48.

Weiss, P. A. (1969). The living system: determinism stratified, in A. Koestler and J. R. Smythies (Eds.), Beyond reductionism: New perspectives in the life sciences (pp. 3-55). Boston: Beacon Press.

Wigner, E. P. (1964). Events, laws of nature, and invariance principles. Science 145, 995-99. 
Wilson, J. (2010). Non-reductive physicalism and degrees of freedom, British Journal for the Philosophy of Science, 61, 279-311.

Wimsatt, W. (1997). Aggregativity: Reductive heuristics for finding emergence, Philosophy of Science, 64 (Proceedings), S372-S384.

Wolfram, S. (2002). A new kind of science. Champaign, IL: Wolfram Media, Inc. 


\section{NOTES}

1. I provide an account of metaphysical reductionism and its formalist assumptions both in an ancient context (Parmenides, Plato, Aristotle) and a modern context (Hume, Kant, Hegel) in Essential Difference (2012). I also explore there the issues treated in this essay more completely and consider what emergence might entail in biological and mental phenomena.

2. Weiss (1969, 21, fn.5) also adopted it fairly early on, noting that Polanyi's view was "crucial" to his own. We also find it more recently in Salthe $(1985,1993)$, Küppers (1992), Stephan (1992), Van Gulick (1993), Emmeche et al. (2000), Christiansen (2000) and Moreno and Umerez (2000). Wilson's (2010) focus on "degrees of freedom" adopts a very similar strategy (without relying on any of this earlier work); her treatment provides a valuable perspective on the idea.

3. I am grateful to a reviewer of this paper for raising a question that led to the preceding clarification of this issue.

4. See the discussion following Proposition 5 of his section on "Dynamics" in his Metaphysical Foundations of Natural Science (1786).

5. The formalist reductionism described here is the same, in principle, as that which Aristotle associated with Plato and Parmenides. A differentia, which Aristotle took to be the substantial principle of form itself, is not a contingent or accidental variation of the possibilities contained within the genus it differentiates (derivable from that genus), but is an actualization of those possibilities that yields an emergent substance. I explain all this more completely in Essential Difference (2012). 
6. I do examine this question more completely in Essential Difference (2012).

7. I am grateful to the two anonymous reviewers of this paper for their detailed comments. The result is a significantly improved version of the original. 